\title{
Effects of depressive symptomatology on cancer-related symptoms during oral oncolytic treatment
}

\author{
Veronica Decker ${ }^{1}$ | Alla Sikorskii ${ }^{2}$ | Charles W. Given ${ }^{3}$ (D) | Barbara A. Given ${ }^{3}$ (D) । \\ Eric Vachon ${ }^{3}$ I John C. Krauss ${ }^{4}$
}

${ }^{1}$ College of Nursing, University of Central Florida, Orlando, FL, United States

${ }^{2}$ Department of Psychiatry and Department of Statistics and Probability, Michigan State University, East Lansing, MI, United States

${ }^{3}$ College of Nursing, Michigan State University, East Lansing, MI, United States

${ }^{4}$ Hematology Oncology, University of Michigan, Ann Arbor, MI, United States

Correspondence

Charles W. Given, PhD, Professor Emeritus, Michigan State University, C346 Bott Building, East Lansing, MI 48824, United States.

Email: givenc@msu.edu

Funding information

National Cancer Institute, Grant/Award Number: 1R01CA162401-01A1

\author{
Abstract \\ Objective: This manuscript assesses association between depressive symptoms and \\ symptoms from cancer and its treatment during the first 12 weeks of a new oral \\ oncolytic treatment.
}

Methods: This secondary analysis used data from a recently completed trial of an intervention to improve adherence to oral oncolytic treatment and manage symptoms. Following the initiation of the new oral oncolytic medication, 272 patients were interviewed at intake and weeks 4,8 , and 12 to assess depressive symptoms, and symptoms from cancer and its treatment. Depressive symptoms were measured using the Center for Epidemiologic Studies-Depression (CES-D20). The summed index of 18 cancer-related and treatment-related symptoms as well as the number of symptoms above threshold at intake, weeks 4,8 , and 12 were related to intake and timevarying CES-D20 using linear mixed effects models.

Results: Depressive symptomatology was a significant predictor of cancer-related and treatment-related symptoms at all-time points, but the strength of this relationship was greatest at the time of oral oncolytic agent initiation and at week 4 . The strength of this relationship was the same for both summed symptom severity index and the number of symptoms above threshold, and using either intake or time-varying CES-D20.

Conclusion: Introducing strategies to treat and manage symptoms of depression along with other symptoms might have added benefits among patients who start a new oral oncolytic treatment and report modest to higher levels of depressive symptoms. Assessments for the impact of strategies to lower depressive symptoms can be taken within the first 4 weeks.

KEYWORDS

cancer, depression, oncology, oral agents, symptoms

\section{1 | BACKGROUND}

Depression occurs in up to $60 \%$ of people with cancer, with prevalence varying based on cancer type and the extent of the disease. ${ }^{1}$
Cancer patients may manifest depression as an ongoing condition, in response to their disease, or as a side effect of treatment, such as the neuropsychiatric effect of chemotherapy. ${ }^{2}$ Many patients suffer from depressive symptoms that are not sufficiently severe to warrant 
a clinical diagnosis of depression ${ }^{3}$ but may increase clinic visits, extended hospitalizations, ${ }^{4,5}$ reduced adherence to treatment, ${ }^{6-8}$ and lower quality-of-life. , $9-11^{-1}$

The relationship between depression and common symptoms/side effects has been studied. ${ }^{12}$ Ciarmalle and Poli ${ }^{13}$ and Uchitomi and colleagues ${ }^{14}$ reported that somatic symptoms such as insomnia and fatigue may either cause depression or be a result of depression. Conclusions from this work are plagued by the use of multiple assessments tools, differing thresholds for identifying depression, ${ }^{15,16}$ provider beliefs that feelings of depression are normal, and their reluctance to diagnose and treat depression. ${ }^{17}$ Further, side effects from treatment and symptoms from other pathologies often overlap, ${ }^{18}$ thereby clouding further the role of depression in symptom experience during cancer treatment.

This work extends this knowledge by determining the associations of depressive symptomatology with symptoms associated with oral oncolytic therapy within the first 12 weeks of initiation. Most studies indicate that patient depression is associated with higher levels of symptom severity, and some studies indicate that depression may intensify the cancer patient's perception of the symptoms. ${ }^{19}$ However, two interrelated issues deserve clarification. First, what is the relationship between patient depressive symptomatology and cancer-related symptoms across time: is the relationship stable, strengthened, or diminished? Second, are reports of summed symptom severity as well as number of symptoms reported at a 4 or higher on a 10-point scale (a standard for a symptom intervention) ${ }^{20}$ equally sensitive to patients' reports of depression as assessed at intake only versus assessments of depression over time? Answers to these questions could inform future symptom management trials by pointing to the importance of considering depressive symptoms as a prognostic factor or a time-varying explanatory variable for patient's cancer-related and treatment-related symptom experience over time. These questions are addressed in this secondary analysis of data from baseline and weeks 4, 8, and 12 collected in a National Cancer Institute funded randomized controlled trial where the aims were to improve adherence to oral oncolytic therapy and to reduce symptom severity.

\section{2 | METHODS}

\section{1 | Study design and sample}

This research was approved by the Institutional Review Board at Michigan State University (IRB\# 13-076 M) and each participating comprehensive cancer center. Patients were recruited between 2013 and 2017 from six $\mathrm{NCl}$-designated Comprehensive Cancer Centers (University of Michigan, Ohio State University, Yale University, Indiana University, Northwestern University, and University of Pittsburgh). Inclusion criteria were as follows: 21 years of age or older, Eastern Cooperative Oncology Group score of 2 or higher or a Karnofsky score of $\geq 50$, able to read and speak English, had a cellular or land line telephone, and new prescription of any one of 28 US Food and Drug Administration (FDA) approved oral oncolytic agents selected according to their FDA approval and emerging use in treating prevalent cancers (Supplemental Table 1 lists the 28 drugs prescribed to consented patients). Patients prescribed preventive and adjuvant medications for breast cancer such as tamoxifen, raloxifene, and aromatase inhibitors were excluded.

Two hundred seventy-two patients newly prescribed one of 28 FDA approved oral oncolytic agents were enrolled, completed the baseline interview, and randomized 1:1 to either the intervention or standard care control arm using a minimization algorithm run from the central study office to ensure concealment. The algorithm balanced trial arms for recruitment location, site of cancer, level of depressive symptoms, oral agent regimen complexity (continuous vs intermittent dosing), and concurrent intravenous chemotherapy. Subsequent interview data were collected via telephone at week 4 (trial midpoint), week 8 (trial endpoint), and week 12 (follow-up). Interviewers were blinded to trial arm assignment. In addition to the three follow-up interviews, both groups received weekly (weeks 2-8) automated voice response system calls. These calls were made at prearranged times and assessed the severity and interference of $18 \mathrm{com}$ mon cancer symptoms.

Participants assigned to the experimental arm who rated a symptom at greater than or equal to 4 were referred to the printed Medication Management and Symptom Management Toolkit (Toolkit) ${ }^{21}$ sent to them following randomization. The Toolkit offered evidence-based interventions for management of 18 symptoms and specific strategies for adherence to oral oncolytic treatment.

Patients in the experimental arm received daily reminder calls to indicate when and how to take their medication and for those drugs with rest periods, when to stop and resume their medication.

The results of the trial indicated that adherence was high and did not differ by trial arm, symptom severity was similar at week 4, lower in the intervention arm at week 8 , and no differences at week $12 .{ }^{22}$ Trial arm assignment was balanced by level of depression controlled for in this secondary analysis of symptoms and depression.

\subsection{Instruments and measures}

\subsection{1 | Depressive symptoms}

The Center for Epidemiologic Studies Depression Scale-20 (CES-D20) was used to evaluate depression at baseline and weeks 4, 8, and 12; higher scores reflect higher levels of depression. ${ }^{23}$ Responses to the 20 items were assessed using a 4-point Likert-type scale ranging from: rarely or none of the time; some of the time; most of the time; and almost all or all of the time. This measure of depressive symptoms in cancer patients is common in the literature. ${ }^{24,25}$ The CES-D20 score of 16 or greater is a screening cut-off for clinical depression. However, a number of different cut-points have been reported in the literature ${ }^{23,26}$ and benchmarked against the Structured Clinical Interview for Depression. ${ }^{26,27}$

We considered the distribution of the CES-D20 scores in the sample and used the cut-off of 8 or higher that corresponds with a T-score of 49.8; the PROMIS T-score of 50 is the mean for the United States general population. ${ }^{28}$ This score also corresponds to the mean in large general population samples that were used to validate the CES-D20. ${ }^{23}$ This cut-off determines how persons above the national average for depressive symptoms may differ from those below this 
national standard with regard to each approach to cancer-related symptom severity.

The impact of patient depressive symptomatology on cancerrelated symptoms at baseline, 4, 8, and 12 weeks was assessed in two ways: by considering baseline depressive symptomatology as a prognostic factor for symptom experience over time; and by considering time-varying depressive symptoms as an explanatory variable for cancer-related symptoms at each time point.

\subsection{2 | Symptom experience}

Eighteen cancer- and treatment-related symptoms were assessed, they are: anxiety; constipation; cough; diarrhea; fatigue; headaches; joint pain; lack of appetite; mouth sores; nausea or vomiting; numbness or tingling especially hands and feet; pain; redness or peeling in hands or feet; shortness of breath; skin rash; sleep disturbance; swelling of hands or feet; and weakness. At intake, 4, 8, and 12 weeks patients were asked if they had experienced each of these symptoms, those reporting "Yes" to each symptom were then asked to rate its severity on a 1 to 9 scale with 1 begin barely noticeable, to a 9 being worst possible. Severity was summarized as (1) summed index ranging from 0 to 162; and (2) number of symptoms scored at a 4 or higher (threshold) at each observation.

\subsection{3 | Use of antidepressants}

Antidepressant therapy was assessed via audit of each patient's medical record which took place at the end of the study. Each patient was then categorized according to whether or not they had been prescribed an anti-depressant medication at intake into the trial.

\subsection{4 | Demographic data and clinical history}

Data on age, sex, ethnicity, education, marital status, income, and employment status were obtained at baseline. Clinical data on cancer diagnosis, stage of cancer, medications for comorbidities, cancer treatments, and complications were obtained from the medical record audit conducted after patients completed the study (week 12) or dropped out. Oral oncolytic medications newly prescribed for patients were collapsed into four classes: kinase inhibitors, cytotoxics, sex hormone inhibitors, and others (Supplemental Table 1).

\section{3 | DATA ANALYSES}

Distributions of the symptom and CES-D20 scores were summarized. Demographic and clinical characteristics of the sample were compared according to the baseline CES-D20 level using t-, chi-square, or Fisher's exact tests as appropriate. To assess the effects of depressive symptomatology of cancer-related symptoms, linear mixed effects (LME) model was fit for four repeated measures of symptom outcomes, summed severity index, and number of symptoms above threshold (one at a time). The explanatory variables were weeks 4, 8, and 12 (entered as a class variable to model potentially nonlinear patterns), trial arm, patient's age and sex, drug category, use of antidepressants, baseline CES-D20 score category ( $\leq 8$ versus $8+$ ), and baseline CES-D20 score by week interaction. The LME model generalizes classical analysis of repeated measures and allows for data missing at random, so that all patients with non-missing symptom and CESD20 scores at baseline were analyzed. The inclusion of the CES-D20 level by time interaction was included to evaluate whether the relationship between depressive symptomatology and cancer-related symptoms changes as time progresses. Least-square means of symptom outcomes according to the levels of the interaction term were output from the LME models, and differences between them according to the levels of baseline CES-D20 were tested. The LME modeling was then repeated with time-varying CES-D20 as a covariate instead of fixed baseline CES-D20.

\section{4 | RESULTS}

\section{1 | Description of sample}

Of the 458 patients screened, 272 consented and completed baseline interviews. Two patients did not complete the CES-D20 at baseline and dropped out following baseline interview; thus, this manuscript is based on $N=270$ patients. Table 1 provides a description of the primary study sample overall and according to CES-D20 level at baseline. Patients were mainly Caucasian (89\%), equal number of men and women, mean age was just over 60 , and $74 \%$ had at least some college education. Audits indicated that, on average, patients took medications for 3.5 comorbid conditions. Cancers were mostly advanced solid tumors, and majority of patients were treated with cytotoxic agents or targeted kinase inhibitors. Patients averaged 3.26 cancerrelated symptoms above threshold at baseline, with mean severity index of 29.09. Both the number of baseline symptoms above threshold, and summed severity index differed significantly according to the CES-D20 level at baseline $(P<.01$, Table 1$)$. Patients with CES-D20 above 8 at baseline were older $(P=.01)$ and greater proportion (32\%) were on anti-depressants compared with patients with baseline CES-D20 of 8 or lower, where $17 \%$ were on anti-depressants. The percent of patients with the CES-D20 above eight declined over time with $45 \%$ at baseline, $35 \%$ at week $4,33 \%$ at week 8 , and $30 \%$ at week 12. The mean CES-D20 in this high group was 17.48 (standard deviation (SD) 7.94) at baseline (Table 1), then 16.72 (SD 8.26) at week 4, 14.58 (SD 5.20) at week 8, and 15.62 (SD 7.04) at week 12.

The strength of association between the CES-D20 at baseline and cancer-related symptoms differed over time; the interaction terms in the LME models were significant for both outcomes of symptom severity $(F(3,647)=8.10, P<.01$ for the interaction term $)$ and number of symptoms above threshold $(F(2,647)=6.04, P<.01)$. The LS means of cancer-related symptom outcomes according to the baseline CESD20 level (Table 2) indicate while differences in cancer-related symptom experience according to the level of CES-D20 were significant at each time point, both mean difference in severity and number of symptoms above threshold decreased over time. While the mean levels of cancer-related symptom measures did not change over time among those with baseline CES-D20 $\leq 8$, there was a significant decrease over time among those with baseline CES-D20 above 8 (Table 2, Figure 1A,C). When time-varying CES-D20 level was used 
TABLE 1 Characteristics of the participants at baseline for the entire sample analyzed, and by the baseline CES-D20 score subgroup

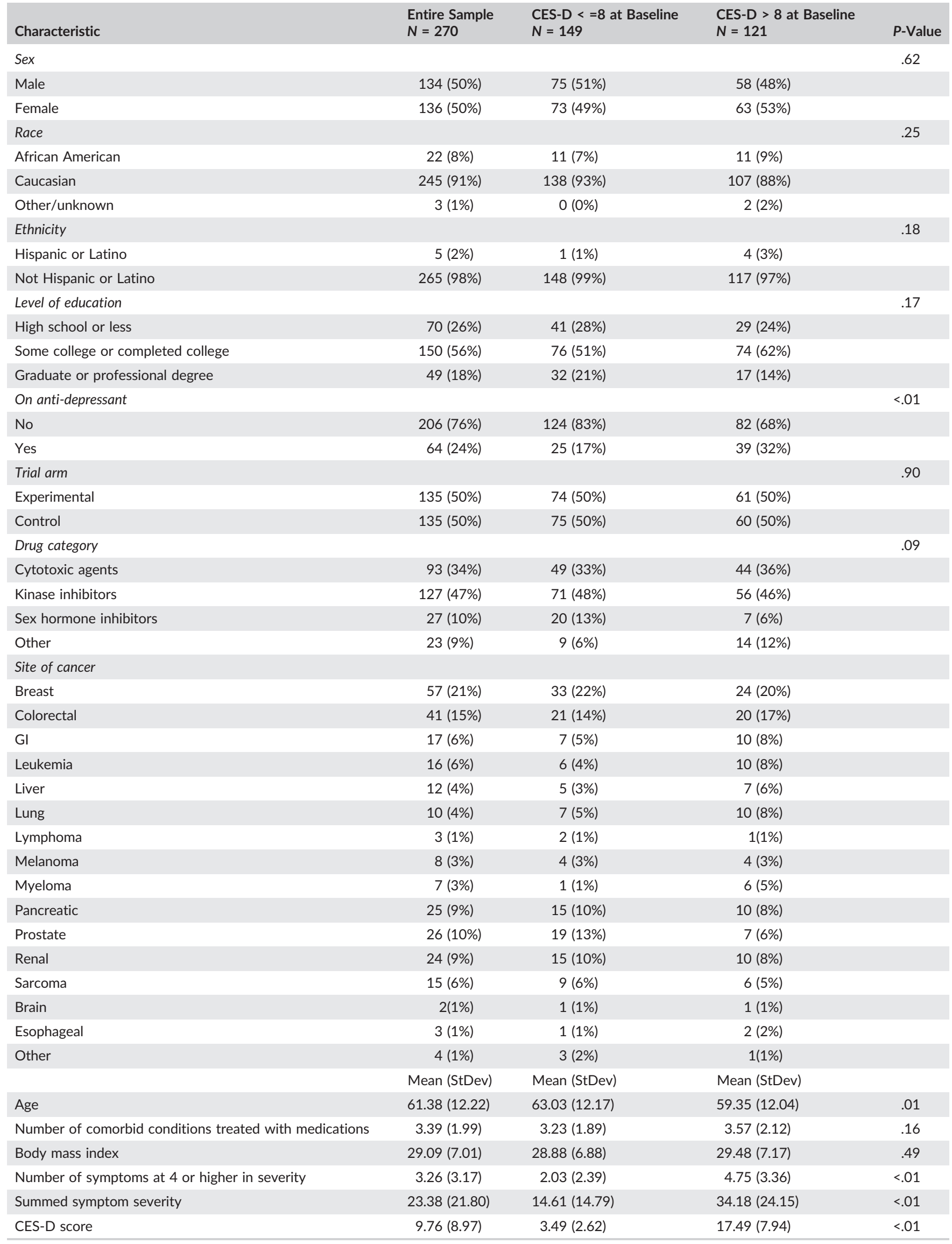


TABLE 2 The least square means (LS) and standard errors (SE) of symptom severity and number of symptoms above threshold by the CES-D20 score at baseline and time

\begin{tabular}{|c|c|c|c|c|}
\hline Time & Outcome & $\begin{array}{l}\text { CES-D20 } \leq 8 \text { at Baseline } \\
\text { LS Mean (SE) }\end{array}$ & $\begin{array}{l}\text { CES-D20 > } 8 \text { at Baseline } \\
\text { LS Mean (SE) }\end{array}$ & Difference $(95 \% \mathrm{Cl}), \mathrm{P}$-Value \\
\hline Baseline & $\begin{array}{l}\text { Symptom severity index } \\
\text { Number of symptoms above threshold }\end{array}$ & $\begin{array}{r}13.37(1.67) \\
1.81(0.25)\end{array}$ & $\begin{array}{r}32.37(1.81) \\
4.46(0.27)\end{array}$ & $\begin{array}{l}-19.00(-23.42,-14.58),<.01 \\
-2.56(-3.30,-1.99),<.01\end{array}$ \\
\hline Week 8 & $\begin{array}{l}\text { Symptom severity index } \\
\text { Number of symptoms above threshold }\end{array}$ & $\begin{array}{r}12.15(1.79) \\
1.49(0.27)\end{array}$ & $\begin{array}{r}25.25(2.03) \\
3.43(0.30)\end{array}$ & $\begin{array}{l}-13.11(-18.08,-8.12),<.01 \\
-1.94(-2.69,-1.20),<.01\end{array}$ \\
\hline
\end{tabular}
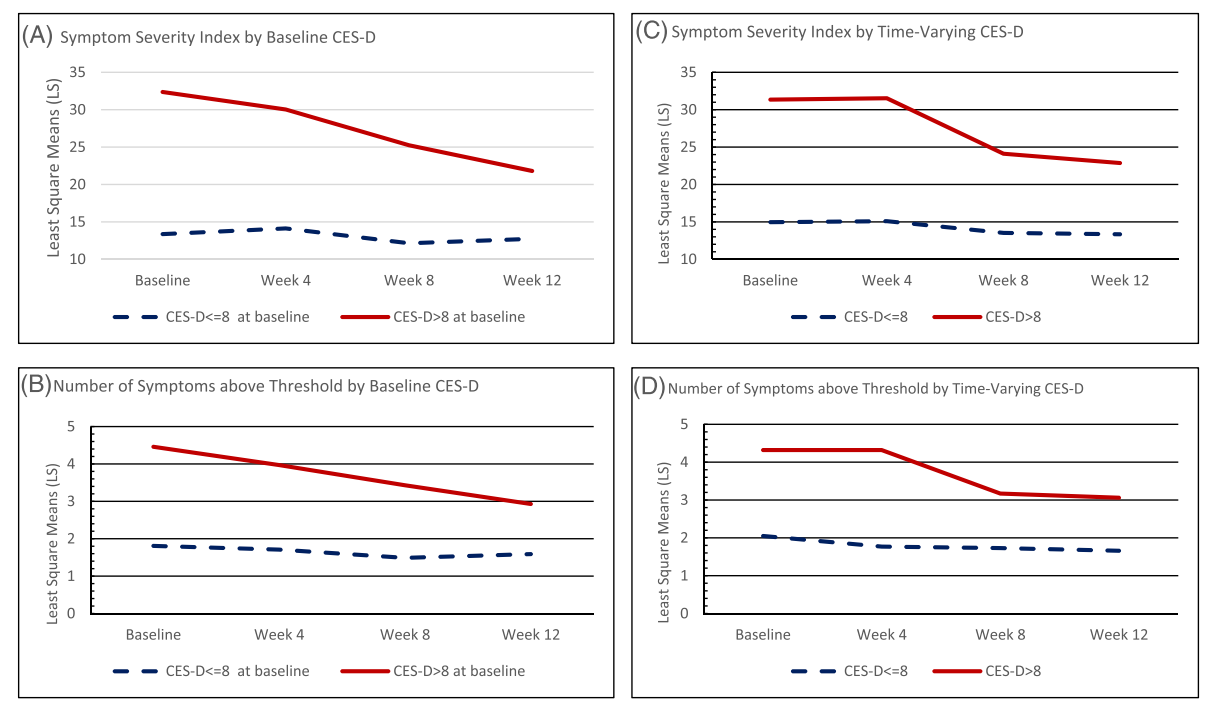

FIGURE 1 Least square means for summed severity and above threshold at baseline and over time by depression scores

TABLE 3 The least square means (LS) and standard errors (SE) of symptom severity and number of symptoms above threshold by time-varying CES-D20 score and time

\begin{tabular}{|c|c|c|c|c|}
\hline Time & Outcome & $\begin{array}{l}\text { CES-D20 } \leq 8 \\
\text { LS Mean (SE) }\end{array}$ & $\begin{array}{l}\text { CES-D20 > } 8 \\
\text { LS Mean (SE) }\end{array}$ & Difference (95\% Cl), P-Value \\
\hline Baseline & Symptom severity index & $14.97(1.53)$ & 31.35 (1.64) & $-16.38(-20.08,-12.69),<.01$ \\
\hline \multirow[t]{2}{*}{ Week 4} & Symptom severity index & $15.10(1.50)$ & $31.53(1.83)$ & $-16.43(-20.21,-12.66),<.01$ \\
\hline & Number of symptoms above threshold & $1.77(0.22)$ & $4.32(0.27)$ & $-2.55(-3.12,-1.99),<.01$ \\
\hline Week 8 & Symptom severity index & $13.53(1.60)$ & $24.12(2.08)$ & $-10.58(-15.11,-6.06),<.01$ \\
\hline \multirow[t]{2}{*}{ Week 12} & Symptom severity index & $13.35(1.64)$ & $22.87(2.01)$ & $-9.53(-13.60,-5.45),<.01$ \\
\hline & Number of symptoms above threshold & $1.66(0.22)$ & $3.06(0.30)$ & $-1.40(-2.01,-0.79),<.01$ \\
\hline
\end{tabular}

as a covariate instead of the fixed baseline CES-D20, the findings were similar (Table 3, Figure 1B,D). Time-varying CES-D20 level was sensitive in pinpointing the time of the decrease (4 weeks) for the explanatory role of depressive symptomatology on cancer-related symptoms. Symptom severity and the number of symptoms above threshold for patients who scored above 8 on the CES-D20 at baseline remained stable for the first 4 weeks, but then decreased during weeks 4 through 8 and reached a plateau for weeks 8 through 12 . Finally, age, sex, drug category, and use of anti-depressants were not predictive of cancer-related symptom experience in any of the models over and above the CES-D20 score.

\section{5 | CONCLUSIONS}

This investigation describes how levels of depressive symptomatology at the onset of oral oncolytic treatment and over 12 weeks differentiate patients according to cancer-related symptoms over time.

A recent review ${ }^{1}$ indicates the prevalence of depressive disorders varies between $5 \%$ and $60 \%$ depending on diagnostic criteria, depressive assessment tools, and type and stage of cancer. The cut-off of 16 on CES-D20 has been used to screen for depression, ${ }^{21,23}$ and in past studies of this team with patients treated with infusion chemotherapy for solid tumor cancers, $34 \%$ of patients scored at or above $16 .{ }^{29}$ In a 
recent study among patients hospitalized for advanced cancer, the rate of depressive symptoms was $29 \%{ }^{30}$ In this sample of patients for whom oral oncolytic treatment often represented the last available line of treatment, only $23 \%$ scored at 16 or higher on the CES-D20. In this report, we used a lower cut-off of 8 on CES-D20 that corresponded to being above or below the national mean for depressive symptoms, according to the cross-walk between the CES-D20 and the PROMIS Depression measure. ${ }^{28}$ Reasons for lower CES-D20 scores in this sample may include a different point in cancer treatment trajectory compared with previous samples on infusion chemotherapy, and prescription of anti-depressants, which were taken by $24 \%$ of patients at the time of initiation of oral oncolytic treatment.

Compared with previous studies with patients on infusion chemotherapy, where summed severity was in the mid 30's, the mean summed index was approximately $23,{ }^{31}$ with the same standard deviation of approximately 23 across similar numbers of symptoms (17 to 18). One reason for lower symptom scores could be a period of no treatment before oral agent initiation.

In this sample, compared with patients who scored 8 or lower on CES-D20, those above 8 had significantly more symptoms above threshold and greater summed symptom severity index at all observations. However, the magnitude of the difference in symptom experience according to the CES-D20 level decreased over time. While the symptom burden remained low among those with the CES-D20 of 8 or lower, both summed severity and number of symptoms at 4 of higher were reduced between the 4 and 8 week observations and reached a plateau between weeks 8 and 12 . What accounts for this decline among those scoring higher on depressive symptomatology remains speculative; the supportive impact of the oral treatment, or relief over continuing to receive treatment all may be explanations. The subgroup of patients with higher CES-D20 became smaller beginning with week 4, possibly because concerns about new treatment were alleviated. ${ }^{32}$ The decline in the CES-D20 by week 4 may explain subsequent decline in symptoms over weeks 4 to 8 , but this explanation could only apply to the results of the analysis with fixed baseline CES-D20 level as a covariate. Since the same reduction in symptoms was seen for time-varying CES-D20 level, it appears that even a modest reduction in the actual CES-D20 score, while still remaining over 8 , could be associated with reduction in other symptoms. The mechanisms by which this occurred are not clear. However, the data point to the value of incorporating interventions focused on depression directly into symptom self-management trials. Specifically, addressing depression near the time of initiation of oral oncolytic agents may make self-management of other symptoms more effective.

\section{1 | Study limitations}

Several limitations deserve mentioning. First, an abiding challenge toward testing the strength and time order of these relationships is the diversity of depression measures, their applications to different sites, stages of cancer, and to treatment modalities. Likely symptoms such as fatigue, pain, and sleep disturbance may contribute to depressive symptoms. Some literature supports the inclusion of somatic items in depression measures, ${ }^{33,34}$ but somatic items are intentionally not included in the PROMIS depression measure. ${ }^{35}$ One of the key issues in choosing items for the depression measurement is that symptom attribution is difficult at best and unreliable at worst. ${ }^{36-38}$ For example, is fatigue due to cancer, its treatment, comorbid conditions, or depression?

Second, these data are confined to only the first 12 weeks of treatment and do not depict the longer trajectory linking the CESD20 and levels of symptoms. Third, results of these analyses point to associations between depressive and other cancer-related symptoms, and there may or may not be a causal relationship. Future work should further examine multiple co-occurring symptoms during this treatment modality. Finally, these data were drawn from a symptom management trial that was successful in lowering symptoms at the 8-week observation compared with the control arm, with no significant differences at the 12-week observation. These observed differences, in part, were mitigated by the fact the arms of the trial were balanced according to level of depression at randomization, and trial arm assignment was controlled for in the present analysis.

\section{2 | Clinical implications}

This work points to the potential value of a cut-off of 8 on the CES-D20 to differentiate the course of symptoms among cancer patients undergoing oral oncolytic treatments. These data could inform behavioral interventions for patients starting oral oncolytic treatment by first introducing strategies to treat and manage symptoms of depression and determining if subsequent self-management approaches toward other symptoms are more effective when depression is addressed. Future work might compare strategies that combine management of depression with other symptoms, with those that focus on depression alone or other cancer-related symptoms alone. From such comparisons, more could be learned about how managing symptoms of depression may reduce the severity of other symptoms. ${ }^{39}$ Further, these comparisons might indicate how soon and to what degree the severity of other symptoms may follow a reduction in depressive symptoms.

\section{ACKNOWLEDGEMENTS}

This work was supported by the National Cancer Institute (1R01CA162401-01A1).

\section{CONFLICT OF INTERESTS}

The authors do not have any potential conflicts of interest, including relevant financial interests, activities, relationships, and affiliations relevant to the subject of this manuscript.

\section{ORCID}

Charles W. Given (i) http://orcid.org/0000-0001-7478-2621

Barbara A. Given (D) http://orcid.org/0000-0002-3971-214X

\section{REFERENCES}

1. Caruso R, Nanni MG, Riba M, et al. Depressive spectrum disorders in cancer: prevalence, risk factors and screening for depression: a critical review. Acta Oncol. 2017;56(2):146-155. 
2. Smith HR. Depression in cancer patients: pathogenesis, implications and treatment (review). Oncol Lett. 2015;9(4):1509-1514.

3. Ganz PA. Monitoring the physical health of cancer survivors: a survivorship-focused medical history. J Clin Oncol. 2006;24(32):5105-5111.

4. Ostuzzi G, Matcham F, Dauchy S, Barbui C, Hotopf M. Antidepressants for the treatment of depression in people with cancer. Cochrane Database Syst Rev. 2015;6. CD011006. doi: https://doi.org/10.1002/ 14651858.CD011006.pub2

5. Prieto JM, Blanch J, Atala J, et al. Psychiatric morbidity and impact on hospital length of stay among hematologic cancer patients receiving stem-cell transplantation. J Clin Oncol. 2002;20(7):1907-1917. https://doi.org/10.1200/JCO.2002.07.101

6. Arrieta O, Angulo LP, Núñez-Valencia C, et al. Association of depression and anxiety on quality of life, treatment adherence, and prognosis in patients with advanced non-small cell lung cancer. Ann Surg Oncol. 2013;20(6):1941-1948. https://doi.org/10.1245/s10434012-2793-5

7. DiMatteo MR, Lepper HS, Croghan TW. Depression is a risk factor for noncompliance with medical treatment: meta-analysis of the effects of anxiety and depression on patient adherence. Arch Intern Med. 2000;160(14):2101-2107.

8. Walker J, Hansen $\mathrm{CH}$, Martin $\mathrm{P}$, et al. Prevalence, associations, and adequacy of treatment of major depression in patients with cancer: a cross-sectional analysis of routinely collected clinical data. Lancet Psychiatry. 2014;1(5):343-350. https://doi.org/10.1016/S22150366(14)70313-X

9. Chan A, Ng TR, Yap KY. Clinically-relevant anticancer-antidepressant drug interactions. Expert Opin Drug Metab Toxicol. 2012;8(2):173-199. https://doi.org/10.1517/17425255.2012.645804

10. Navari RM, Brenner MC, Wilson MN. Treatment of depressive symptoms in patients with early stage breast cancer undergoing adjuvant therapy. Breast Cancer Res Treat. 2008;112(1):197-201. https://doi. org/10.1007/s10549-007-9841-z

11. Pinquart M, Duberstein PR. Depression and cancer mortality: a metaanalysis. Psychol Med. 2010;40(11):1797-1810. https://doi.org/ $10.1017 /$ S0033291709992285

12. Miaskowski C, Dunn L, Ritchie C, et al. Latent class analysis reveals distinct subgroups of patients based on symptom occurrence and demographic and clinical characteristics. J Pain Symptom Manage. 2015;50(1):28-37.

13. Ciaramella A, Poli P. Assessment of depression among cancer patients: the role of pain, cancer type and treatment. Psychooncology. 2001;10(2):156-165. https://doi.org/10.1002/pon.505

14. Uchitomi Y, Mikami I, Nagai K, Nishiwaki Y, Akechi T, Okamura H. Depression and psychological distress in patients during the year after curative resection of non-small-cell lung cancer. J Clin Oncol. 2003;21(1):69-71. https://doi.org/10.1200/JCO.2003.12.139

15. Saracino RM, Weinberger MI, Roth AJ, Hurria A, Nelson CJ. Assessing depression in a geriatric cancer population. Psychooncology. 2017;26(10):1484-1490. https://doi.org/10.1002/pon.4160

16. Wakefield C, Butow PN, Aaronson NA, Hack TF, Hubert-Williams NJ, Jacobsen PB. Patient-reported depression measures in cancer: a meta-review. Lancet Psychiatry. 2015;2(7):635-647. https://doi.org/ 10.1016/S2215-0366(15)00168-6

17. Pasquini $M$, Biondi $M$. Depression in cancer patients: a critical review. Clin Pract Epidemiol Ment Health. 2007;3(1):2.

18. Li M, Fitzgerald P, Rodin G. Evidence-based treatment of depression in patient with cancer. J Clin Oncol. 2012;30(11):1187-1196. https://doi. org/10.1200/JCO.2011.39.7372

19. Hermelink $\mathrm{K}$, Kuchenhoff $\mathrm{H}$, Untch $\mathrm{M}$, et al. Two different sides of 'chemobrain': determinates and nondeterminates of self-perceived cognitive dysfunction in a prospective, randomized, multicenter study. Psychooncology. 2010;19(12):1321-1328. https://doi.org/10.1002/ pon.1695
20. National Comprehensive Cancer Network. NCCN clinical practice guidelines in Oncology, Version 2.2016; 2016. Fort Washington, PA: National Comprehensive Cancer Network; c2018 [Cited 2018 June 25]. https://www.nccn.org/professionals/physician_gls/default.aspx

21. Given BA, Given CW, Majeske C. Medication Management and Symptom Management Toolkit. East Lansing MI: Michigan State University; 2013.

22. Sikorskii A, Given CW, Given BA, et al. An automated intervention did not improve adherence to oral oncolytic agents while managing symptoms: results from a two-arm randomized controlled trial. J Pain Symptom Manage. 2018. August Epub before print. https://doi.org/ 10.1016/j.jpainsymman.2018.07.021

23. Radloff LS. The CES-D20 Scale: a self-report depression scale for research in the general population. Appl Psychol Measur. 1977;1(3):385-401.

24. Carleton RN, Thibodeau MA, Teale MJ, et al. The center for epidemiologic studies depression scale: a review with a theoretical and empirical examination of item content and factor structure. PLoS One. 2013;8(3): e58067. https://doi.org/10.1371/journal.pone.0058067

25. Segrin C, Badger T, Dorros SM, Meek P, Lopez AM. Interdependent anxiety and psychological distress in women with breast cancer and their partners. Psychooncology. 2007;16(7):634-643.

26. Vilagut G, Forero CG, Barbaglia G, Alonso J. Screening for depression in the general population with the Center for Epidemiologic Studies Depression (CES-D20): a systematic review with metaanalysis. PLoS One. 2016;11(5):e0155431. https://doi.org/10.1371/ journal.pone.0155431

27. First MB, Spitzer RL, Gibbon M, Williams JBW. Structured Clinical Interview for DSM-IV-TR Axis I Disorders, Research Version, Non-Patient Edition. Biometric Research Department, New York State Psychiatric Institute; 2002.

28. PROsetta Stone Linking Tables. [website]. Illinois: David Cella, Ph.D., Department of Medical Social Sciences at the Feinberg School of Medicine, Northwestern University [Cited 2018 June 25]. http://www. prosettastone.org/LinkingTables1/Pages/default.aspx

29. Given B, Given C, Sikorskii A, et al. Establishing mild, moderate and severe scores for cancer related symptoms: how consistent and clinically meaningful are interference based severity cut points? J Pain Symptom Manage. 2008;35(2):126-135.

30. Nipp RD, El-Jawahri A, Moran SM, et al. The relationship between physical and psychological symptoms and health care utilization in hospitalized patients with advanced cancer. Cancer. 2017;123(23):4720-4727. https://doi.org/10.1002/cncr.30912.

31. Sikorskii A, Given C, Given B, et al. Symptom management for cancer patients: a trial comparing two multimodal interventions. J Pain Symptom Manage. 2007;34(3):253-264.

32. Sikorskii A, Given C, Given B, et al. Do treatment patterns alter beliefs cancer patients hold regarding oral oncolytic agents? Psychooncology. 2018;27(3):1005-1012. https://doi.org/10.1002/pon.4606

33. Clover K, Lambert SD, Oldmeadow C, et al. PROMIS depression measures perform similarly to legacy measures relative to a structured diagnostic interview for depression in cancer patients. Qual Life Res. 2018;27(5):1357-1367. https://doi.org/10.1007/s11136-018-1803-x

34. van Wilgen CP, Dijkstra PU, Stewart RE, Ranchor AV, Roodenburg JL. Measuring somatic symptoms with the CES-D2O to assess depression in cancer patients after treatment: comparison among patients with oral/oropharyngeal, gynecological, colorectal, and breast cancer. Psychosomatics. 2006;47(6):465-470.

35. Health measures [homepage on the internet]. Illinois: Northwestern University [Cited 2018 June 25]. Healthmeasures.net

36. Cleeland CS, Sloan JA, Cella D, et al. CPRO (assessing the symptoms of cancer using patient-reported outcomes) multisymptom task force. Recommendations for including multiple symptoms as endpoints in cancer clinical trials: a report from the ASCPRO (assessing the symptoms of cancer using patient-reported outcomes) multisymptom task 
force. Cancer. 2013;119(2):411-420. https://doi.org/10.1002/ cncr. 27744

37. Cleeland CS, Zhao F, Chang VT, et al. The symptom burden of cancer: evidence for a core set of cancer-related and treatment-related symptoms from the Eastern Cooperative Oncology Group Symptom Outcomes and Practice Patterns study. Cancer. 2013;119(24):4333-4340. https://doi.org/10.1002/cncr.28376

38. Hillman SL, Mandrekar SJ, Bot B, et al. Evaluation of the value of attribution in the interpretation of adverse event data: a North Central Cancer Treatment Group and American College of Surgeons Oncology Group investigation. J Clin Oncol. 2010;28(18):3002-3007. https://doi. org/10.1200/JCO.2009.27.4282

39. Voils $\mathrm{Cl}$, King HA, Maciejewski ML, Allen KD, Yancy WS Jr, Shaffer JA. Approaches for informing optimal dose of behavioral interventions. Ann Behav Med. 2014;48(3):392-401. https://doi.org/10.1007/ s12160-014-9618-7

\section{SUPPORTING INFORMATION}

Additional supporting information may be found online in the Supporting Information section at the end of the article.

How to cite this article: Decker V, Sikorskii A, Given CW, Given BA, Vachon E, Krauss JC. Effects of depressive symptomatology on cancer-related symptoms during oral oncolytic treatment. Psycho-Oncology. 2019;28:99-106. https://doi. org/10.1002/pon.4916 